\title{
Estrutura a Termo da Taxa de Juros e Imunização: Novas Perspectivas na Gestão do Risco de Taxa de Juros em Fundo de Pensão(1)
}

\author{
Sérgio Jurandyr Machado \\ Luis Felipe Jacques da Motta
}

\begin{abstract}
RESUMO
O termo imunização denota a construção de uma carteira de títulos de forma a torná-la imune a variações nas taxas de juros. No caso dos fundos de pensão, o objetivo da imunização é distribuir os recebimentos intermediários e finais dos ativos de acordo com a previsão para o fluxo de pagamentos dos benefícios. Nesse sentido, o artigo busca comparar o desempenho de duas alternativas de imunização ao método tradicional que restringe o gerenciamento do risco de taxa de juros à compatibilização da duration de ativos e passivos. As carteiras de títulos foram obtidas a partir de métodos de programação linear, nos quais as restrições são decorrentes da alternativa de imunização escolhida. O indicador de desempenho utilizado foi o valor em risco (VaR), aqui definido como o menor valor presente da diferença entre ativos e passivos do fundo de pensão, para um dado nível de confiança. O VaR foi calculado a partir da descrição probabilística da evolução das taxas de juros, obtida por meio de uma simulação. Os resultados permitem não apenas avalizar a estratégia mais eficiente, como também explicitar a superioridade do desempenho das duas alternativas em relação ao método tradicional, auxiliando a criação de valor na indústria de previdência complementar.
\end{abstract}

Palavras-chave: imunização; estrutura a termo; valor em risco; fundo de pensão.

\begin{abstract}
Immunization is defined as the investment in assets in such a way that the fixed income portfolio is immune to a change in interest rates. In the special case of pension funds, immunization seeks the distribution of the cash inflows in accordance with the outflows represented by the fund's liabilities. The article compares two alternative methods of immunization against the traditional durationmatching strategy. Both portfolios were obtained as a result of linear programming problems, where the choice of the immunization strategy led to the restrictions imposed. The measure used to assess the performance of each portfolio was the value at risk (VaR), here defined as the difference of the assets and liabilities present values. A simulation for the Brazilian term structure of interest rates was conducted in order to provide the possible outcomes for future rates, making it possible to compute the value at risk of the actual assets against the long-term liabilities. The article provides the basis for selecting the most appropriate method for immunization and also demonstrates the superiority of both alternatives against the traditional duration-matching strategy, helping to devise a consistent investment policy for pension funds.
\end{abstract}

Key words: immunization; term structure; value at risk; pension fund. 


\section{INTRODUÇÃO}

A criação de valor na indústria de previdência complementar está associada à capacidade do gestor em prover o beneficiário do plano de uma alocação eficiente de capital. Após o período de diferimento, ou seja, findo o prazo de pagamento das contribuições para o fundo, a alocação eficiente pode ser compreendida como a busca pela minimização da probabilidade de insolvência do fundo de pensão, de forma a garantir o pagamento dos benefícios estipulados em contrato.

Partindo-se da premissa de que o fluxo de benefícios pode ser descrito de forma análoga ao fluxo de um título de renda fixa, cria-se um incentivo natural para a utilização das reservas técnicas - referentes aos benefícios concedidos na compra desse tipo de instrumento financeiro. Entretanto, títulos de renda fixa estão sujeitos ao risco de taxa de juros, estimulando uma gestão ativa no sentido de minimizá-lo.

Se o montante e o momento de ocorrência dos dispêndios podem ser antecipados com exatidão, a estratégia menos arriscada para a entidade de previdência consiste em aplicar o valor presente desses dispêndios em títulos sem cupom com data de vencimento idênticas às dos pagamentos a serem efetuados. Ao adotar esse método para a aplicação dos recursos disponíveis, o gestor estará construindo uma carteira dedicada. As carteiras dedicadas são, por natureza, isentas do risco de taxa de juros.

Ainda que seja possível adotar a estratégia de dedicação para horizontes de investimento de curta duração, o mercado brasileiro de renda fixa praticamente inviabiliza esse tipo de operação no médio e longo prazo. As Letras do Tesouro Nacional - LTN, títulos prefixados e sem pagamentos intermediários, possuem prazo médio de 7,83 meses (janeiro/2004). Ademais, praticamente todo o fluxo de saída de um fundo de pensão está indexado à variação anual de um índice de preços. O Tesouro Nacional não emite títulos com cláusula de atualização monetária sem o pagamento de cupons intermediários.

Uma opção consiste em se criar um porta-fólio de títulos com cupom que minimize a probabilidade de perdas associadas a alterações paralelas na curva da taxa de juros. Essa estratégia, denominada imunização tradicional, busca tornar uma carteira de títulos de renda fixa imune aos efeitos de pequenas modificações paralelas da curva da taxa de juros. 
Figura 1: Evolução da Estrutura a Termo

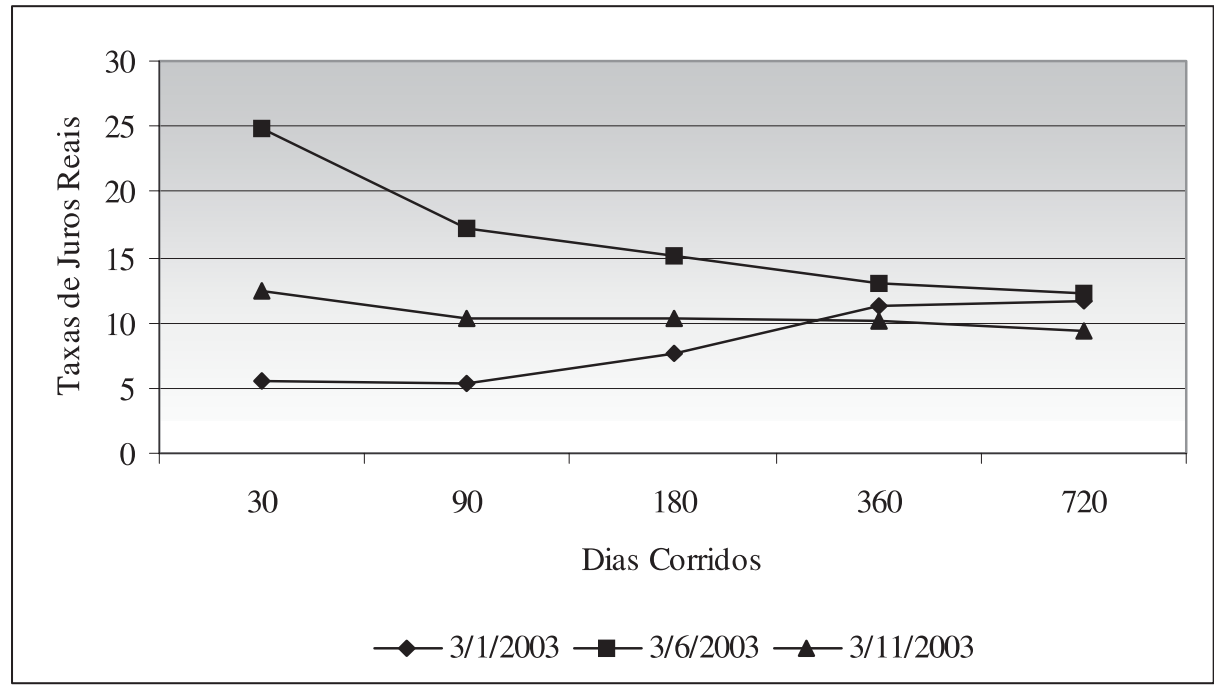

Fonte: Bolsa de Mercadorias e Futuros.

Entretanto, conforme descrito na Figura 1, a assunção de que a curva da taxa de juros irá modificar-se apenas por meio de movimentos paralelos é demasiado ingênua. Ademais, existem formas alternativas de se igualar a duration de ativos e passivos. Pode-se adotar uma estratégia em que o vencimento dos títulos que compõem a carteira estão distantes da duration do passivo ou, alternativamente, uma estratégia na qual o vencimento dos títulos que compõem a carteira se aproximam da duration do passivo. A primeira estratégia é mais arriscada, uma vez que os cupons estarão expostos por um prazo maior ao risco de reinvestimento (risco de imunização).

A teoria de imunização evoluiu no sentido de minimizar esse risco de reinvestimento, tendo sido criadas alternativas de administração de risco baseadas em choques arbitrários sobre a estrutura a termo. Nesse sentido, o objetivo do artigo é comparar o desempenho de duas dessas alternativas de imunização à estratégia de imunização tradicional.

O indicador de desempenho será o valor em risco (VaR), aqui definido como o menor valor presente da diferença entre ativos e passivos do fundo, para um dado nível de confiança (99\%). O valor presente da diferença entre ativos e passivos foi escolhido porque retrata a capacidade de um fundo de pensão em satisfazer as obrigações impostas pelo plano de benefícios acordado entre participantes e patrocinadores. 
Para tanto, é necessário que se exponha a carteira de investimento da fundação a uma série de choques arbitrários da taxa de juros obtidos a partir de modelos de estrutura a termo. Por simplificação, o passivo será reduzido a cinco saídas de caixa, 12, 24, 36, 48 e 60 meses após a data de construção das carteiras (novembro de 2003). O artigo encontra-se organizado da seguinte maneira: a segunda parte trata do risco de imunização; a terceira parte discute as peculiaridades da modelagem do mercado de renda fixa; a quarta parte descreve uma taxonomia básica dos modelos de estrutura a termo; a quinta parte apresenta alguns conceitos básicos da metodologia para o cálculo do VaR; a sexta parte descreve as carteiras ótimas obtidas; e a sétima, o cômputo do VaR e os resultados da análise.

\section{Risco de IMUNIZAÇÃo}

A literatura sobre gerenciamento do risco de taxa de juros é vasta, estando associada, basicamente, à especificação de medidas alternativas de duration. A cada modelo particular de comportamento futuro da estrutura a termo corresponderá uma medida de sensibilidade dos preços dos títulos. Soto (2004) realiza uma análise pormenorizada desses modelos e conclui que a diferença entre os métodos se restringe basicamente ao número de fatores e à forma como esses influenciam a curva de juros, reduzindo a imunização a uma questão de dimensão (onde cada dimensão é equivalente a um fator) de duration a ser alcançada.

Esse tipo de metodologia restringe a imunização a um movimento particular da curva de taxa de juros. Se a estrutura a termo futura for diferente da prevista por esse movimento, o porta-fólio estará sujeito a perdas. Ademais, a imunização de uma carteira a choques de dimensão superior a um está condicionada à existência da possibilidade de venda a descoberto de títulos.

Fong e Vasicek (1984) foram os primeiros a propor uma estratégia de administração de risco para uma carteira exposta a choques arbitrários da taxa de juros, sem a necessidade de se realizarem operações a descoberto. A hipótese básica do modelo consiste na divisão do risco de taxa de juros em dois componentes básicos: a magnitude da alteração sofrida pela estrutura a termo e o tipo de título (ou conjunto de títulos) exposto a essa modificação. O segundo termo representa uma medida de risco de imunização. A minimização desse risco busca reduzir a variabilidade do valor de um título em função de movimentos aleatórios das taxas de juros. 
Ao testar a hipótese, Fong e Vasicek demonstram que o risco de imunização pode ser descrito pela variável $M^{2}=\frac{\sum_{i}^{n}\left(t_{i}-D\right)^{2} P V\left(C F_{i}\right)}{\sum_{i}^{n} P V\left(C F_{i}\right)}$, onde $D$ representa a duration do título (ou o horizonte de investimento), $t_{i}$ é o prazo do fluxo $i$ e $P V\left(C F_{i}\right)$ é o valor presente do fluxo $i$.

Trata-se, portanto, de uma medida de dispersão que tenderá a zero na medida em que a data de recebimento dos cupons se aproximar da duration da carteira. De fato, $M^{2}$ será igual a zero se a carteira for composta apenas por um título sem cupom. A estratégia de gerenciamento conjunto de ativos e passivos consistirá, portanto, em minimizar $M^{2}$ para uma dada duration do passivo. De acordo com Fabozzi (2000), na imunização de carteiras com múltiplos horizontes de investimento, o piso da dispersão dos ativos não será zero, mas sim o valor da dispersão do passivo.

Recentemente, Balbás e Ibáñez (1998) propuseram uma nova medida para o risco de imunização. Os autores introduzem o conceito de condição de imunização fraca $^{(2)}$. Esta é verificada na existência de pelo menos um título $i$ no porta-fólio que, em decorrência de um choque $k$ na estrutura a termo, seja capaz de produzir um retorno maior ou igual ao retorno esperado.

Assim, $\frac{V i(k)}{P i} \geq R$, onde $V i(k)$ representa o retorno do título $i$ na ocorrência do choque $k, P i$ o preço do título $i$ e $R$ o retorno esperado. A condição pode ser interpretada da seguinte forma: um investidor que tenha certeza da ocorrência de um determinado choque $k$ irá comprar um título cuja rentabilidade esperada não será comprometida pela alteração na curva de juros.

Suponha-se agora que exista um subconjunto de choques para os quais a condição de imunização fraca não se verifica. Nesse caso, o risco da estratégia de imunização pode ser calculado a partir das perdas ocasionadas pela ocorrência de tais choques e a minimização do risco de imunização estará associada à minimização dessas perdas. Para tanto os autores sugerem a adoção de uma medida do risco de imunização $\tilde{N}=\frac{\sum_{i}^{n}\left|t_{i}-D\right| P V\left(C F_{i}\right)}{\sum_{i}^{n} P V\left(C F_{i}\right)}$.

Embora os dois modelos partam de uma mesma premissa, a da ocorrência de choques arbitrários sobre a curva de taxa de juros, a carteira de ativos é exposta 
a diferentes extensões de choques, o que justifica a diferença entre as medidas de dispersão apresentadas.

\section{Modelagem no Mercado de Juros}

A estrutura a termo da taxa de juros (ETTJ) é definida por Almeida (2001, p. 1) como "o mapa que relaciona o termo de um empréstimo à taxa de juros embutida no mesmo". A análise da eficácia dos métodos de imunização parte do pressuposto de que é possível modelar as variações futuras da ETTJ de forma crível.

Um modelo de estrutura a termo da taxa de juros constitui-se em uma descrição probabilística da sua evolução futura. A incerteza decorre do fato de que a informação disponível em determinado momento do tempo não é suficiente para descrever, de forma perfeita, a estrutura a termo futura. A análise quantitativa representada pelo modelo será, portanto, um espelho dessa incerteza.

A modelagem de instrumentos de renda fixa possui características próprias que a distinguem da modelagem de outros ativos financeiros. De acordo com Vieira Neto (1999), essas idiossincrasias podem ser resumidas nos seguintes pontos.

a) Tendência de reversão à média: as taxas de juros reais tendem a se mover em direção a uma média de longo prazo. Tal tendência encontra respaldo na teoria econômica, na medida em que uma política monetária restritiva tende a se tornar neutra (ou expansionista) após a ocorrência do impacto negativo desejado sobre o nível de produto e renda. De forma análoga, políticas monetárias expansionistas tendem a ser seguidas por políticas neutras ou restritivas.

b) Descrição do processo estocástico da curva de juros em vários pontos e não somente em determinado prazo: ao contrário de outros ativos financeiros, os títulos de renda fixa que realizam pagamentos intermediários (cupons) são afetados por taxas de juros de prazos distintos que, normalmente, apresentam valor também distinto.

c) Multiplicidade de volatilidades: as volatilidades nos diversos pontos da estrutura a termo (vértices) costumam diferir; e as correlações entre os diferentes vértices de taxa de juros não são perfeitas, decrescendo na medida em que aumenta a distância entre eles.

O mercado brasileiro reproduz, de forma similar aos mercados internacionais, essas mesmas características. Entretanto, no que diz respeito à reversão à média, 
deve-se salientar que, ao contrário dos países de mercado financeiro desenvolvido, cujas taxas de médio e longo prazo não são facilmente contaminadas pelos problemas de curto prazo, no Brasil é praticamente impossível identificar e medir a taxa de juros real de equilíbrio de longo prazo.

\section{Uma Proposta de Taxonomia}

A construção de uma taxonomia objetiva uma melhor compreensão das principais características dos modelos que tentam descrever a estrutura a termo, distinguindo e classificando-os em subcategorias. Cabe salientar que o exercício se restringe apenas aos seis modelos apresentados a seguir, embora o número de métodos disponibilizados na literatura seja infinitamente superior.

\section{Quadro 1: Taxonomia dos Modelos de Estrutura a Termo}

\begin{tabular}{|c|c|c|}
\hline Tipo de Modelo & Fator Único & Multifatorial \\
\hline Equilíbrio & VASICEK (1977) & \\
\hline Não-Arbitragem & CIR (1985) & HJM (1992) \\
& HULLEE (1986) & DUFFIE-KAN (1996) \\
\hline
\end{tabular}

Fonte: adaptado de Vieira Neto (1999).

Basicamente, os modelos de ETTJ se dividem em modelos de equilíbrio (Cox, Ingersoll, \& Ross, 1985; Vasicek, 1977) e de não-arbitragem (Duffie \& Kan, 1996; Heath, Jarrow, \& Morton, 1992; Ho \& Lee, 1986; Hull \& White, 1990).

Os modelos distinguem-se, ainda, pelo número de fatores associados às alterações na ETTJ. Nos unifatoriais, toda a estrutura a termo é função de uma única variável ou fator, a taxa de juros de curto prazo. Essa é definida como a taxa de desconto que se aplica a um período infinitesimal em um dado ponto $t$ do tempo. Daí o fato de também ser chamada de taxa infinitesimal de curto prazo ou taxa instantânea.

A associação a apenas uma fonte de incerteza não é demasiadamente restritiva. Hull (2000) afirma que o método unifatorial implica todas as taxas movendo-se na mesma direção, mas não necessariamente na mesma intensidade. A estrutura a termo nem sempre tem a mesma forma; amplo espectro de curvas de taxas de juros pode ocorrer sob a perspectiva desse método. 
Nos modelos de equilíbrio, também conhecidos como endógenos, a curva de juros em dado tempo té derivada analiticamente a partir dos parâmetros definidos e não há garantia de que ela será idêntica à curva de mercado efetivamente observada. Em um artigo pioneiro, Vasicek (1977) explicita as bases dos modelos de equilíbrio unifatoriais, descrevendo o processo para a taxa instantânea de juros a partir da seguinte equação diferencial estocástica:

$$
d r=a(b-r) d t+V d z
$$

onde $a, b$ e VEãa constantes que descrevem a taxa de reversão à média, a média e o desvio-padrão, respectivamente, da taxa de juros de curto prazo e $d z$ um processo de Wiener básico, no qual $z$ tem distribuição normal com média zero e variância $t$.

Intuitivamente, pode-se compreender o processo descrito por Vasicek como o impacto sobre a taxa de juros de curto prazo decorrente da variação infinitesimal do tempo e da variância associada $V d z$. Sempre que $r$ for maior que $b$, o termo $a(b-r)$ será negativo, impactando negativamente a taxa de curto-prazo. Analogamente, sempre que $r$ for menor que $b$, o impacto será positivo. Dessa forma, temos um processo de reversão à média.

O ponto fraco do método proposto por Vasicek decorre do fato de que o modelo pode gerar taxas reais de curto prazo negativas, o que dificilmente ocorrerá na realidade. Nesse sentido, Cox et al. (1985) propõem um procedimento alternativo, em que o processo para a taxa de juros instantânea é descrito por $d r=a(b-r) d t+\sigma \sqrt{r} d z$. O componente representado pela raiz de $r$ impede que as taxas de juro de curto prazo se tornem negativas.

O aspecto endógeno do procedimento adotado nos métodos de equilíbrio é normalmente apresentado pelos analistas financeiros como o responsável pela maior utilização dos modelos de não-arbitragem ou exógenos. Nestes, o procedimento é calibrado de forma a ajustar a estrutura a termo inicial à curva de juros efetivamente observada no mercado.

Pioneiro na linha dos modelos de não-arbitragem, o método de Ho e Lee (1986) transforma a curva de juros prevalecente no mercado em um input do processo. Nesse sentido, o modelo consubstancia-se no primeiro método coerente com os princípios da não-arbitragem. A versão contínua do processo para a taxa de juros de curto prazo passa a ser descrito por:

$d r=\frac{\partial \theta(t)}{\partial t} d t+\sigma d z$ 
onde $T(t)$ é uma função determinista escolhida de forma a possibilitar que o modelo seja capaz de gerar, na data inicial, uma estrutura a termo idêntica à observada no mercado. Em contrapartida, o procedimento proposto por Ho e Lee desconsidera uma eventual tendência de reversão à média da taxa de juros de curto prazo.

Hull e White (1990) exploram essa deficiência do modelo de Ho e Lee, propondo uma extensão do modelo de Vasicek:

$d r=a\left(\frac{\theta(t)}{a}-r\right) d t+\sigma d z$

Portanto, a taxa de juros de curto prazo tende à média $\frac{\theta(t)}{a}$ a uma taxa $a$. No modelo de Hull e White, diferentemente do proposto por Ho e Lee, a análise contempla um espectro mais amplo de volatilidade, já que esta é afetada concomitantemente pela constante de reversão à média $a$ e pelo desviopadrão $\sigma$.

Atualmente, existe uma tendência em se estender a análise da estrutura a termo por meio da utilização de modelos multifatoriais. Heath et al. (1992), Duffie e Kan (1996) e vários outros autores têm buscado incrementar a precisão dos modelos propostos por meio da inserção de novos fatores de incerteza.

Essa multiplicidade de dimensões será tanto mais eficiente quanto menor a correlação entre as variâncias observadas em pontos distintos da estrutura a termo. Os modelos multifatoriais estão normalmente associados a uma implementação mais lenta e dispendiosa e, portanto, o analista deve examinar criteriosamente o custo-benefício existente, especialmente no caso de as taxas apresentarem alto grau de correlação.

Dada a taxonomia e as características básicas dos modelos descritos, o gestor de recursos poderia indagar se realmente existe um método que deva ser eleito como o mais apropriado para o exame do comportamento futuro das taxas de juros. Vários critérios podem ser identificados como necessários a um modelo de estrutura a termo. A princípio, o método deve ter significado econômico, ser implementável e aproximar de forma bastante precisa os preços observados no momento da análise. Ocorre que não apenas um, mas vários modelos citados preenchem esses requisitos básicos e, portanto, são elegíveis.

Apesar de toda a evolução da teoria financeira sobre o assunto, não há garantia de que o processo estocástico escolhido será capaz de predizer, de 
forma precisa, os movimentos futuros da estrutura a termo. Portanto, a escolha recairá sobre aquele procedimento passível de implementação que, na opinião do analista financeiro, melhor se adaptar aos movimentos futuros mais prováveis. Para efeito deste artigo, o modelo escolhido para a simulação da estrutura a termo foi o proposto por Ho e Lee (1986). Além da simples implementação, o modelo é condizente com a realidade de mercado e, conforme salientado anteriormente, negligencia o processo de reversão à média. Assim, não há necessidade de se trabalhar com o conceito de taxa de juros real de equilíbrio de longo prazo, dada a dificuldade no cômputo desse dado para o mercado brasileiro.

\section{O Valor em Risco}

Em sua forma geral, o valor em risco (VaR) mede o risco de mercado - a forma como mudanças nos preços dos ativos financeiros afetam o valor das posições em aberto de uma carteira de investimentos, em determinado horizonte de tempo, para dado nível de confiança. O VaR representa uma tentativa de prover os gestores de risco de um valor específico que sumarie a perda máxima associada a uma carteira. No caso específico de instrumentos de renda fixa, o VaR calculado será uma estimativa do risco de taxa de juros.

Basicamente, existem três formas para se calcular o VaR.

a) Método analítico: dada a hipótese de que os retornos sejam normais e independente e identicamente distribuídos, pode-se computar o valor de mercado de uma carteira (e, por conseguinte, o VaR) como função linear de seus parâmetros: média, variâncias e correlações. Nesse caso, o valor de risco é representado pelo limite inferior do intervalo de confiança para o retorno da carteira. Assim, por exemplo, para o nível de significância de 5\%, o VaR será igual ao retorno médio menos 1,65 vezes o desvio-padrão da carteira.

b) Método de simulação histórica: a partir da distribuição amostral dos retornos nos últimos $x$ dias, calcula-se o percentil correspondente ao nível de significância desejado. Apesar do nome, não há simulação envolvida, sendo utilizada apenas a distribuição empírica dos retornos passados da carteira de ativos.

c) VaR obtido via simulação: a partir de processos estocásticos que delineiem a movimentação das variáveis financeiras associadas à carteira, são 
simuladas trajetórias de preço para todos os ativos integrantes do portafólio, gerando uma distribuição de retornos que será utilizada para o cômputo do VaR.

Os três métodos apresentam vantagens e desvantagens examinadas detalhadamente em Jorion (1998). Para efeito desse artigo, o VaR foi calculado a partir de uma simulação, utilizando-se o seguinte procedimento:

1) Utilizando-se o modelo de Ho e Lee (1986), foi obtida uma série de taxas de curto prazo, denotando a evolução futura das taxas de juros.

2) De posse dessas taxas foi possível calcular uma série de prováveis valores presentes, tanto dos ativos quanto do passivo do fundo de pensão.

3) Criou-se uma nova série composta pela diferença entre os valores presentes e, dado o nível de confiança preestabelecido (99\%), calculouse o VaR.

\section{Construindo Carteiras Imunizadas}

Suponha que para a obtenção das carteiras imunizadas, estejam disponíveis os seguintes instrumentos financeiros indexados ao índice geral de preços do mercado (IGP-M) em 03/11/2003:

Tabela 1: Características dos Títulos Públicos e Privados

\begin{tabular}{c|c|r|r}
\hline Título & Vencimento & Preço (em R\$) & \multicolumn{1}{c}{ Duration (em dias) } \\
\hline NTN-C & $1 / 7 / 2005$ & $1.537,36$ & 399,94 \\
\hline NTN-C & $1 / 4 / 2008$ & $1.413,96$ & 986,51 \\
\hline Debênture SABESP & $1 / 3 / 2007$ & $14.431,29$ & 489,33 \\
\hline Debênture COPEL & $1 / 3 / 2007$ & $14.581,90$ & 678,69 \\
\hline
\end{tabular}

Fonte: Andima e Bovespa.

Suponha, ainda, que determinado fundo de pensão possua um passivo (indexado ao IGP-M) com as características descritas na Tabela 2: 
Tabela 2: Características do Passivo

\begin{tabular}{c|c}
\hline Data & Pagamentos (valores em R\$) \\
\hline $1 / 11 / 2004$ & $70.000 .000,00$ \\
\hline $1 / 11 / 2005$ & $70.000 .000,00$ \\
\hline $1 / 11 / 2006$ & $70.000 .000,00$ \\
\hline $1 / 11 / 2007$ & $70.000 .000,00$ \\
\hline $1 / 11 / 2008$ & $70.000 .000,00$ \\
\hline Valor Presente em 03/11/2003 & $270.254 .947,03$ \\
\hline Duration $($ em dias) & 309,36 \\
\hline Dispersão $\tilde{\mathrm{N}}(\mathrm{em} \text { dias })^{2}$ & $127.078,92$ \\
\hline Dispersão $\mathbf{M}^{2}\left(\mathrm{em}\right.$ dias $\left.^{2}\right)$ &
\end{tabular}

Fonte: elaboração dos autores.

A construção das carteiras pressupõe que haverá total liquidez para os instrumentos utilizados, ou seja, os títulos poderão ser adquiridos no mercado secundário pelos preços descritos na Tabela 1. Cabe salientar que foram desconsiderados os custos de transação envolvidos, além dos impostos incidentes sobre as operações. A diferença no risco de crédito dos títulos públicos e privados está refletida nas taxas de desconto distintas utilizadas para cômputo dos preços.

Como existe uma infinidade de combinações desses títulos que viabilizaria a imunização, é necessário realizar uma otimização. Para uma dada função objetivo - que estará relacionada à medida de risco $M^{2}$ ou $\tilde{N}$, tem-se um problema típico de programação matemática, que buscará minimizar a função objetivo, dada uma série de restrições. As variáveis de decisão são representadas por $x_{1}$ (percentual de NTN-C com vencimento em 01/07/2005), $x_{2}$ (percentual de NTN-C com vencimento em 01/04/2008), $x_{3}$ (percentual de debênture Sabesp com vencimento em 01/03/2007) e $x_{4}$ (percentual de debênture Copel com vencimento em 01/03/2007).

Algebricamente, o problema de programação linear pode ser descrito da seguinte forma: 


$$
\text { MINIMIZAR } \frac{\sum_{i}^{n}\left(t_{i}-D\right)^{2} P V\left(C F_{i}\right)}{\sum_{i}^{n} P V\left(C F_{i}\right)} \text { ou } \frac{\sum_{i}^{n}\left|t_{i}-D\right| P V\left(C F_{i}\right)}{\sum_{i}^{n} P V\left(C F_{i}\right)}
$$

\section{SUJEITO A}

$$
\begin{aligned}
& D_{A}=D_{P} \\
& \sum_{i, j}^{n} P V\left(C F_{i}\right) x_{j}=\sum_{p}^{n} P V\left(C F_{P}\right) \\
& x_{1}+x_{2}+x_{3}+x_{4}=1 \\
& x_{1}>0 ; x_{2}>0 ; x_{3}>0 ; x_{4}>0 \\
& x_{3} \leq 0,2 ; x_{4} \leq 0,2
\end{aligned}
$$

A função objetivo depende da escolha da medida de risco de imunização, se $M^{2}$ ou $\tilde{N}$. As restrições (4) e (5) obrigam que a duration e o valor presente do ativo sejam iguais a do passivo, respectivamente. A restrição (6) busca garantir que o somatório dos percentuais dos títulos em carteira seja igual a $100 \%$. A restrição (7) impossibilita a realização de venda a descoberto de qualquer um dos títulos que integra a carteira. Finalmente, a restrição (8) limita o percentual de aplicação em debêntures de emissão de companhia aberta a 20\% do total da carteira (resolução CMN n ${ }^{\circ} 3121 / 2003$ ).

Adotada essa sistemática, têm-se as seguintes carteiras ótimas em 01/11/2003:

Tabela 3: Carteira Imunizada $\tilde{N}$

\begin{tabular}{c|c|r|r}
\hline Descrição & Vencimento & \multicolumn{1}{|c|}{ \% da Carteira } & Valor de Mercado (R\$) \\
\hline NTN-C & $1 / 7 / 2005$ & 18,85 & $50.947 .940,19$ \\
\hline NTN-C & $1 / 4 / 2008$ & 41,42 & $111.942 .276,14$ \\
\hline Debênture SABESP & $1 / 3 / 2007$ & 20,00 & $54.050 .980,33$ \\
\hline Debênture COPEL & $1 / 3 / 2007$ & 19,73 & $53.313 .750,37$ \\
\hline Total & & 100,00 & $270.254 .947,03$ \\
\hline
\end{tabular}

Fonte: elaboração dos autores. 
Tabela 4: Carteira imunizada $M^{2}$

\begin{tabular}{c|c|r|r}
\hline Descrição & Vencimento & \multicolumn{1}{|c|}{ \% da Carteira } & \multicolumn{1}{|c}{ Valor de Mercado (R\$) } \\
\hline NTN-C & $1 / 7 / 2005$ & 31,56 & $85.299 .762,49$ \\
\hline NTN-C & $1 / 4 / 2008$ & 50,25 & $135.809 .790,65$ \\
\hline Debênture SABESP & $1 / 3 / 2007$ & 15,64 & $42.281 .891,67$ \\
\hline Debênture COPEL & $1 / 3 / 2007$ & 2,55 & $6.863 .502,21$ \\
\hline Total & & 100,00 & $270.254 .947,03$ \\
\hline
\end{tabular}

Fonte: elaboração dos autores.

\section{Mensurando a Eficiência de uma Carteira Imunizada}

Formatadas as carteiras imunizadas, deve-se testar a sua eficiência na redução dos riscos associados às taxas de juros. A questão do VaR da diferença entre ativos e passivos adquire especial importância a partir da publicação da Resolução MPS/CGPC nq6, de 30 de outubro de 2003, que regulamenta a portabilidade.

De acordo com a resolução,

entende-se por portabilidade o instituto que faculta ao participante transferir os recursos financeiros correspondentes ao seu direito acumulado para outro plano de benefícios de caráter previdenciário operado por entidade de previdência complementar ou sociedade seguradora autorizada a operar o referido plano.

O direito acumulado para fins de portabilidade irá impor às fundações uma gestão de risco ainda mais eficiente, sob pena de elas mesmas incorrerem em grandes perdas financeiras, por ocasião do eventual exercício da portabilidade por parte dos integrantes do plano.

Conforme descrito anteriormente, o VaR será obtido a partir de uma simulação. A distribuição dos cenários se baseia no modelo estocástico de Ho e Lee (1986) e pode ser verificada no apêndice $I^{(3)}$. Definidos os cenários para as taxas de juros, pode-se calcular os preços para todos os ativos integrantes da carteira, gerando uma distribuição de retornos que será utilizada para o cômputo do VaR. A estrutura a termo inicial é representada pelas taxas de mercado para os contratos a termo para troca de rentabilidade (Swap DI x IGP-M) em 03/11/2003. A diferença entre a taxa de desconto do título público e do título privado (spread) foi fixada em $4,02 \%$ a.a para todos os vértices, taxa idêntica à verificada nas negociações efetuadas no mercado secundário nessa data. 


\section{Figura 2: Estrutura a Termo}

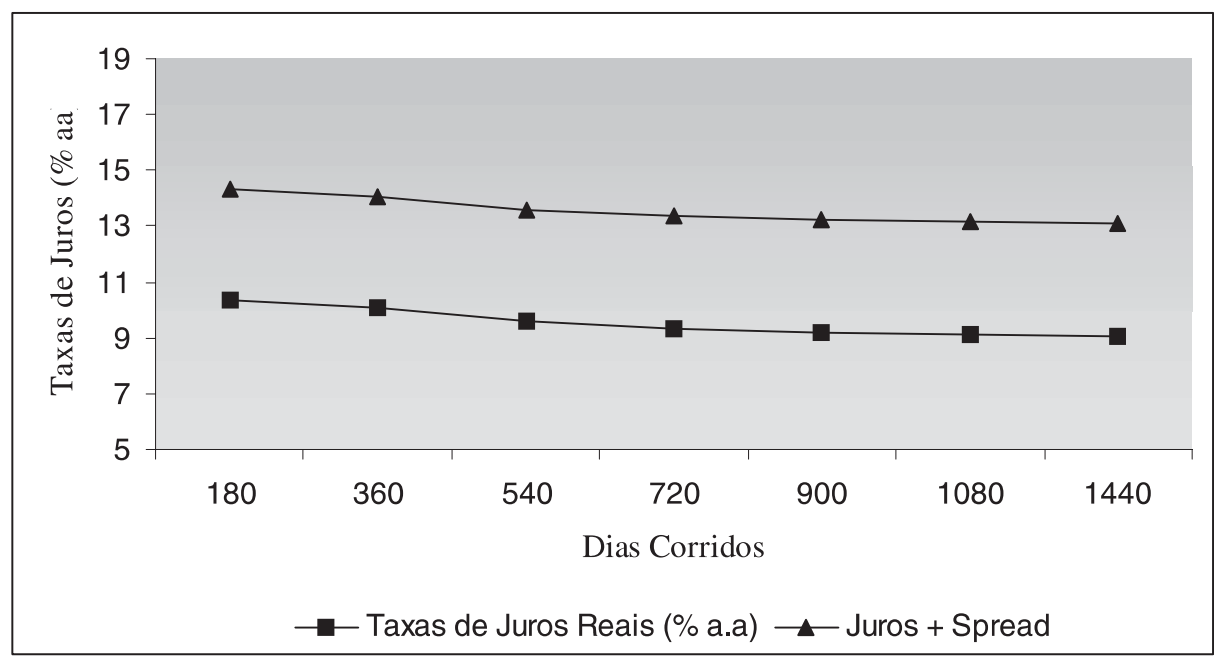

Fonte: Bolsa de Mercadorias e Futuros.

A Tabela 5 traz os resultados referentes às duas carteiras imunizadas e, ainda, a uma carteira obtida a partir da imunização tradicional, na qual apenas a duration do ativo foi igualada a do passivo, negligenciando a questão da dispersão ${ }^{(4)}$. A estratégia de imunização de Balbás e Ibáñez (1998) se mostrou a mais eficiente:

Tabela 5: Valor em Risco

\begin{tabular}{c|c}
\hline Estratégia & VaR (99\%) \\
\hline Carteira imunizada $\tilde{N}$ & $(986.100,13)$ \\
\hline Carteira imunizada $\mathrm{M}^{2}$ & $(1.429 .935,66)$ \\
\hline Imunização Tradicional & $(1.529 .855,45)$ \\
\hline
\end{tabular}

Fonte: elaboração dos autores.

Cabe salientar que estes resultados foram obtidos a partir da utilização de uma volatilidade de aproximadamente $2,0 \%$ a.a para as taxas de juros reais. Trata-se de perspectiva otimista que contempla uma maior estabilidade para o mercado brasileiro nos próximos anos. Entretanto, caso o gestor opte por um cenário mais conservador, é bastante provável que as carteiras imunizadas que minimizam a dispersão apresentem um desempenho ainda mais significativo em relação à imunização tradicional. 


\section{Conclusão}

Até recentemente, o fluxo de pagamentos de parcela significativa dos fundos de pensão era inferior ao montante recebido dos beneficiários a título de contribuição. Atualmente, a situação se inverteu na maioria das fundações; e estratégias como a imunização adquirem importância especial. Num futuro próximo, é possível que tais estratégias venham a desempenhar papel ainda mais importante, uma vez que a previsibilidade do fluxo de saídas aumentará na mesma proporção do incremento do número de benefícios concedidos por tempo predeterminado.

Este estudo apresentou uma evidência direta de que o modelo que se utiliza da medida de risco $\tilde{N}$ foi o mais eficaz no gerenciamento do risco de taxa de juros de um fundo de pensão. Não obstante, as duas alternativas que englobam a questão da dispersão obtiveram desempenho superior ao da imunização tradicional, o que comprova a importância do risco de imunização sobre o desempenho da carteira e, por conseguinte, a necessidade de incluí-lo no gerenciamento de riscos associados a carteiras de renda fixa.

A escolha do processo gerador das estruturas a termo e da volatilidade associada influenciam o resultado final. Trata-se de uma opção do gestor, que deve fazê-lo de modo a tentar retratar os cenários futuros que, em sua opinião, tenham a maior probabilidade de ocorrência.

A estratégia de imunização viabiliza, ainda, a gestão ativa dos recursos restantes do fundo de forma não necessariamente correlacionada ao perfil do passivo. $\mathrm{O}$ percentual de ativos a imunizar e os fatores que influenciam tal decisão constituem objeto de relevância para análise futura.

\section{Artigo recebido em 13.10.2004. Aprovado em 01.03.2005.}

\section{NOTAS}

\footnotetext{
${ }^{1}$ Uma versão preliminar desse artigo foi apresentada no XXVIII ENANPAD, 2004.

${ }^{2}$ Weak immunization condition, no original.

${ }^{3}$ Uma árvore de taxa de juros consubstancia-se em uma representação aproximada do processo estocástico em tempo discreto. Nesse sentido, utilizou-se o software DerivaGem, disponível em Hull (2000). Por simplificação, o exercício contemplou apenas cinco passos. A probabilidade de ocorrência de cada uma dessas taxas é distinta, embora esse fato tenha sido desconsiderado na análise. As taxas intermediárias foram obtidas por meio de uma interpolação linear.

${ }^{4}$ Os instrumentos financeiros utilizados são os mesmos (Tabela 1), embora a composição da carteira seja obviamente diferente.
} 


\section{Referencias Bibliográficas}

Almeida, C. I. R. (2001).

Estimação, teste e aplicações em mercados emergentes: a estrutura a termo da taxa de juros, Tese de Doutorado, Pontifícia Universidade Católica do Rio de Janeiro, RJ, Brasil.

Balbás, A., \&

Ibáñez, A. (1998, December).

When can you immunize a bond portfolio? Journal of Banking \& Finance, 22(12), 1571-1595.

Cox, J. C.,

Ingersoll, J. E., \&

Ross, S. A. (1985).

A theory of the term structure of interest rates, Econometrica, 53, 385407.

Duffie, D., \&

Kan, R. (1996, October).

A yield-factor model of interest rates, Mathematical Finance, 6(4), 379-406.

Fabozzi, F. J. (2000).

Bond markets, analysis and strategies. New Jersey: Prentice Hall International.

Fong, H. G., \&

Vasicek, O. A. (1984, December).

A risk minimizing strategy for portfolio immunization. The Journal of Finance, 39(5), 1541-1546.

Heath, D.,

Jarrow, R., \&

Morton, A. (1992, January).

Bond pricing and the term structure of interest rates: a new methodology for contingent claims valuation, Econometrica, 60(1), 77-105.
Ho, T. S. Y., \&

Lee, S. B. (1986, December).

Term structure movements and pricing interest rate contingent claims. Journal of Finance, 41(5), 1011-1029.

Hull, J., \&

White, A. (1990, March).

Valuing derivative securities using explicit finite difference method. Journal of Financial and Quantitative Analysis, 25(1), 87-100.

Hull, J. (2000).

Options, futures and other derivatives. New Jersey: Prentice-Hall International.

Jorion, P. (1998).

Value at risk. São Paulo: BM\&F.

Soto, G. M. (2004, May).

Duration models and irr management: a question of dimensions? Journal of Banking \& Finance, 28(5), 10891110.

Vasicek, O.A. (1977, November). An equilibrium characterization of the term structure. Journal of Financial Economics, 5(2), 177-188.

Vieira Neto, C.A. (1999).

Modelagem da estrutura a termo da taxa de juros e avaliação de contratos derivativos, Tese de Doutorado, Universidade de São Paulo, São Paulo, SP, Brasil. 


\section{APÉNDICE 1}

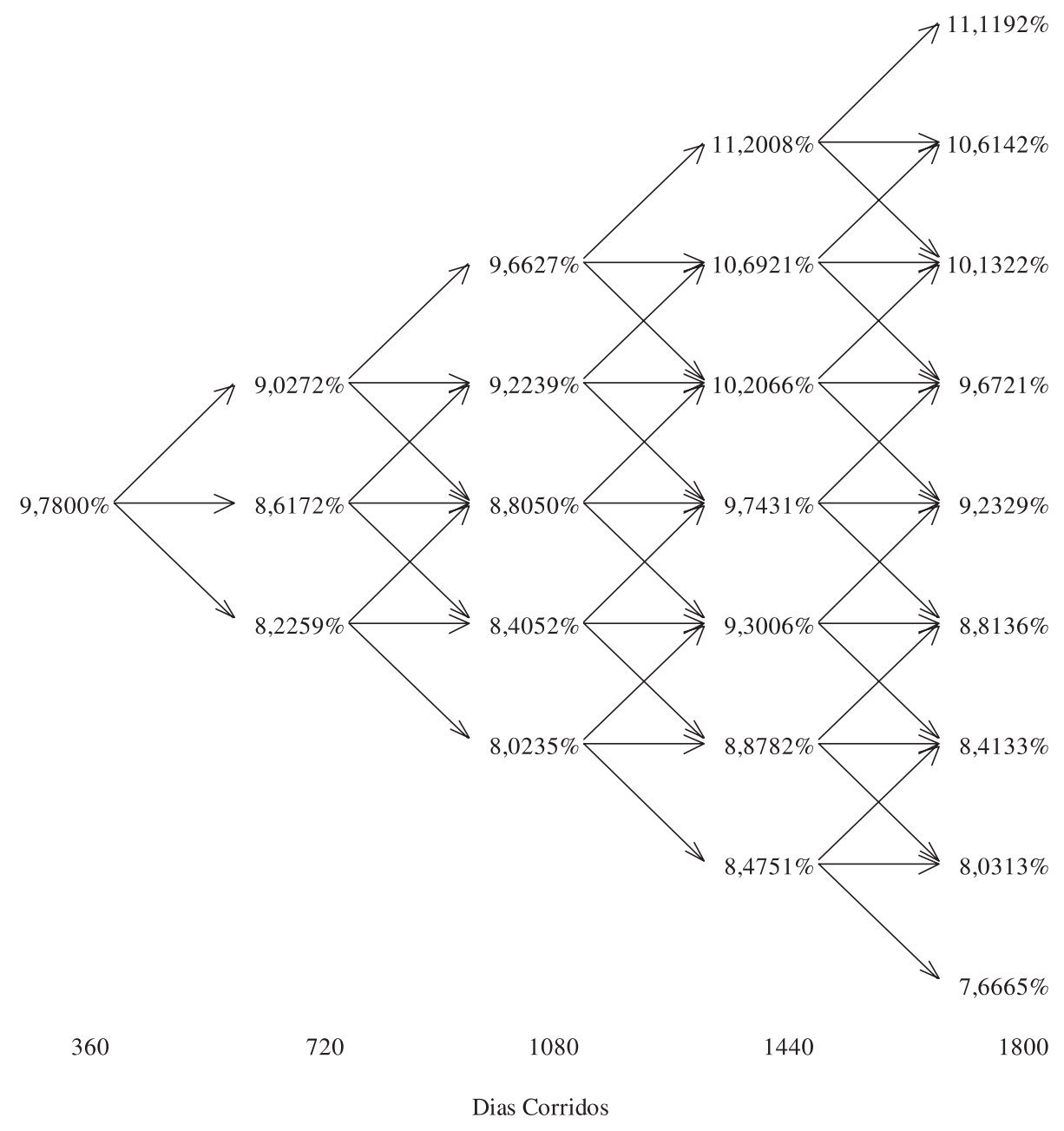

